\title{
CLINICAL COMPARISON OF BLADDER CONTRACTILITY \\ PARAMETERS CALCULATED FROM ISOMETRIC \\ CONTRACTIONS AND PRESSURE-FLOW STUDIES
}

\author{
R. VAN MASTRIGT, PH.D. \\ D. J. GRIFFITHS, PH.D.
}

From the Departments of Urology and Biological

and Medical Physics, Erasmus University,

Rotterdam, the Netherlands

\begin{abstract}
Parameters describing the contractility of the urinary bladder can be calculated from both isometric contractions and pressure-flow studies. The first method has the advantage of making very little demand either on the patient or on the urodynamicist, and the disadvantage of yielding a parameter that is not clearly related to muscle physiology. The second method demands more from both patient and experimenter but yields straightforward parameters. For a group of 86 patients with mixed pathologic findings, a correlation between the two types of parameters was demonstrated, showing that both methods test, at least partly, the same mechanism.
\end{abstract}

A contractility parameter is an objective measure of the ability of a muscle to shorten and/or to develop force. ${ }^{1}$ Two types of contractility parameters can be distinguished, those calculated from isometric contractions, and those calculated from measurements made during shortening of the muscle. ${ }^{2}$ For the urinary bladder, both types have been proposed.

From an isometric contraction a force-velocity relation can be calculated using Hill's $^{3}$ model with an assumed series elasticity. ${ }^{4}$ In only about 16 per cent of the measured contractions, however, can the force-velocity relation, obtained in this way, be fitted with a hyperbolic curve to yield unique contractility parameters. ${ }^{5}$ An alternative method of analysis is to fit a phase plot of the contraction (a plot of the rate of rise of force as a function of force itself) with a straight line of assumed slope. ${ }^{6}$ This method can be applied to all clinically measured isometric contractions. It has the advantage of being very easy to carry out, since isometric contractions can be recorded during a routine cystometry procedure without extra patient load and with minimal extra effort for the urodynamicist. In a study in vitro a strong correlation between a parameter calculated in this way and the maximal contraction velocity of the muscle was found. ${ }^{7}$ The calculated parameter, however, bears no direct relation to muscle physiology, and its usefulness remains to be demonstrated.

From a recording of pressure and flow during micturition a plot of the contraction velocity of the urinary bladder as a function of detrusor pressure can be made. ${ }^{8}$ In about 9 per cent of the pressure-flow studies such a plot traces out part of a hyperbolic pressure-velocity curve (the total-bladder equivalent of the forcevelocity relationship of a muscle strip), and can be fitted with such a curve. This method yields contractility parameters directly related to muscle physiology, but it requires a full pressure-flow study and can be applied only to a small percentage of these studies. 

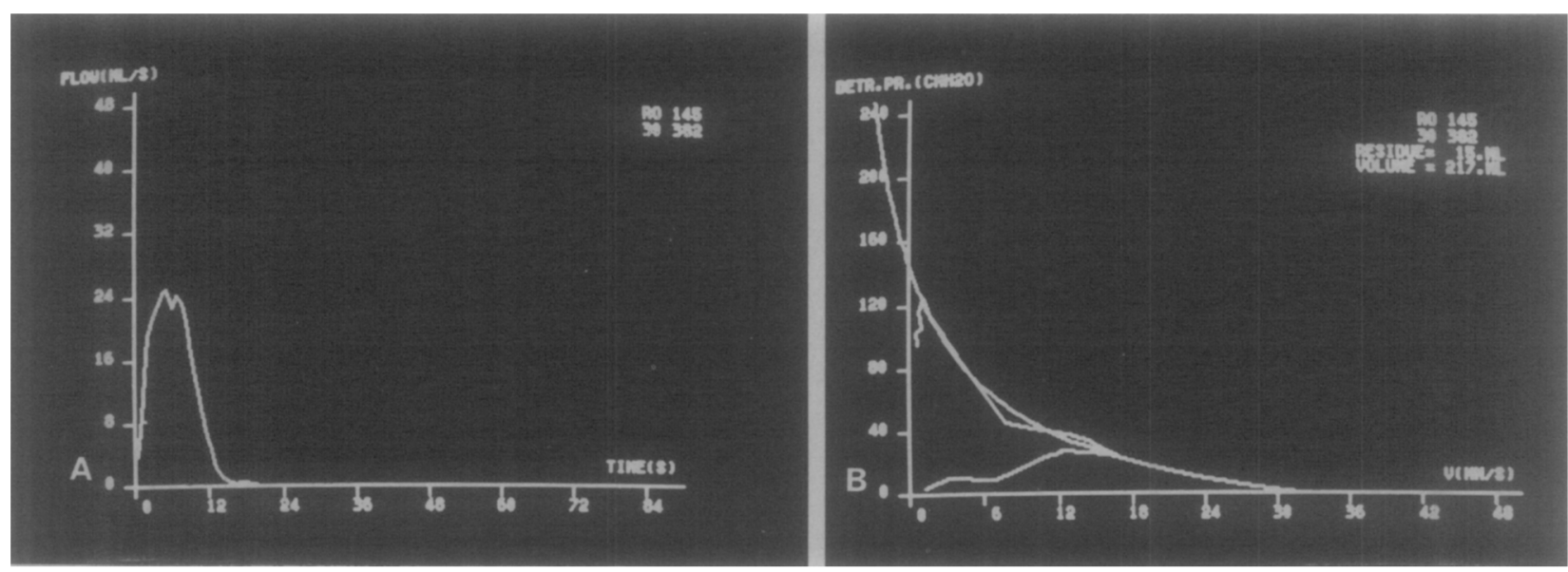

Ficure 1. (A) Flow rate as a function of time during pressure-flow study, and (B) plot of detrusor pressure as a function of contraction velocity calculated from pressure-flow study (A). Part of curve was fitted with hyperbolic pressure-velocity curve with parameters pmax $=145 \mathrm{~cm} \mathrm{H} \mathrm{H}_{2} \mathrm{O}$ and vmax $=32 \mathrm{~mm} / \mathrm{s}$.

The present investigation was performed to compare contractility parameters determined in these two different ways. If a relationship between these parameters can be shown, the general applicability and patient friendliness of the first method might be combined with the physiologic significance of the second.

\section{Material and Methods}

A group of 231 patients, adults and children, with mixed pathologic findings, underwent three (children) or two (adults) consecutive pressure-flow studies. Details of procedure and apparatus have been described previously. ${ }^{\circ} \mathrm{De}-$ trusor pressure and flow during micturition as well as the isometric pressure rise before the onset of micturition were stored by a digital computer,$^{10}$ connected to the urodynamic setup via a telephone line."

Figure 1A shows a measured flow curve. From these pressure-flow studies, a plot of contraction velocity as a function of detrusor pressure was calculated, ${ }^{9}$ taking into account both the residual urine left in the bladder and the delay time between the pressure and flow signals due to the distance between the urinary tract and the flowmeter. In 86 patients one or more of the calculated plots apparently traced out part of the theoretical, hyperbolic pressurevelocity relationship:

$$
(p+a) \cdot(v+b)=(p \max +a) \cdot b
$$

where: $p$ is detrusor pressure; $v$ is contraction velocity; pmax is isovolumetric pressure; and a and $\mathrm{b}$ are parameters. ${ }^{9}$ Introducing a preset curvature $\mathrm{a} / \mathrm{pmax}=0.25^{12}$ yields:

$(p+p \max / 4) \cdot(v+v \max / 4)=5 / 16 \cdot p \max \cdot v \max (2)$ where: $\operatorname{vmax}(=4 \cdot b)$ is the maximum velocity of contraction.

In these 86 cases such a curve was fitted to the data by manually adjusting the intercepts of the curve on the pressure and velocity axes. Figure $1 \mathrm{~B}$ shows a curve which corresponds to data in Figure 1A. From each fitted pressure-contraction velocity relationship, the parameters vmax (the intercept on the velocity axis) and pmax (the intercept on the pressure axis) were therefore obtained. To try to characterize the entire fitted curve with one parameter, vmax and pmax were combined to yield the parameter $w$, which has the dimension of power per surface area. A multiplier of 5/16 was introduced so that $\mathrm{w}$ is equal to the right-hand side of equation (2):

$$
w=5 / 16 \cdot \operatorname{pmax} * v \max
$$

From the isometric pressure rise immediately preceding the successfully fitted pressure-flow relations, the corresponding rise in circumferential force was calculated (Fig. 2A); a phase plot was made and fitted with a straight line with preset slope..$^{67}$ This yielded the parameter $\mathrm{U}$, the extrapolated maximum rate of force rise before micturition starts (Fig. 2B). A few contractions which showed no detectable pressure rise were not analyzed.

In patients for whom more than one pressure-flow relation could be fitted, the three parameters calculated from this relationship and 


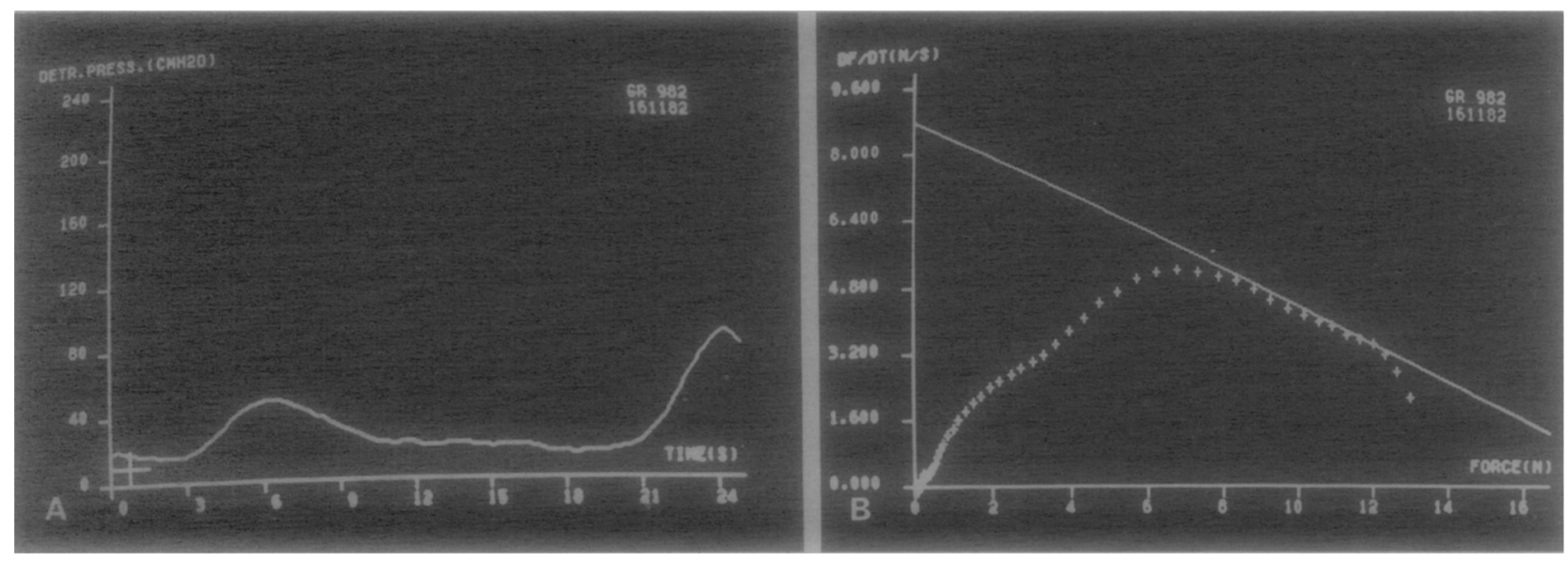

FIGURE 2. (A) Recording of detrusor pressure as a function of time during last twenty-five seconds before onset of micturition; part of curve between nineteenth and twenty-fifth seconds was analyzed as isometric contraction. (B) Phase plot (rate of rise of force as a function of force) calculated from isometric contraction (A). Part of curve was fitted with straight line with preset slope, giving value of $8.8 \mathrm{~N} / \mathrm{s}$ for parameter $U$.

the parameter $\mathrm{U}$ calculated from the preceding isometric contraction were averaged.

Each micturition took place at the bladder volume at which the patient reported severe urgency. This volume was considered as an approximation to the bladder capacity. Since contraction velocity is expected to be proportional

TABLE I. Averages and standard deviations (between parentheses) of contractility parameters calculated from pressure-flow studies and isometric contractions, and bladder volume at which these were measured

\begin{tabular}{llrc}
\hline Parameter & \multicolumn{2}{l}{ Value $(\mathbf{n}=86)$} & Units \\
\hline vinax & 26 & $(8.7)$ & $\mathrm{mm} / \mathrm{s}$ \\
$\mathrm{pmax}$ & 112 & $(55)$ & $\mathrm{cm} \mathrm{H} \mathrm{O}_{2}$ \\
$\mathrm{w}$ & 89 & $(48)$ & $\mathrm{N} / \mathrm{ms}$ \\
$\mathrm{vmax} / \mathrm{L}$ & 0.11 & $(.04)$ & $/ \mathrm{s}$ \\
$\mathrm{U}$ & 8.8 & $(4.7)$ & $\mathrm{N} / \mathrm{s}$ \\
$\mathrm{U} / \mathrm{L}$ & 37 & $(19)$ & $\mathrm{N} / \mathrm{ms}$ \\
Volume & 262 & $(183)$ & $\mathrm{ml}$ \\
\hline
\end{tabular}

TABLE II. Correlations of contractility parameters determined from pressure-flow studies and isometric contractions, with bladder volume, an approximation to the bladder capacity

\begin{tabular}{lc}
\hline Parameter & Corr With Volume \\
\hline vmax & $0.42^{*}$ \\
pmax & 0.24 \\
w & 0.06 \\
$\mathrm{U}$ & $0.42^{*}$ \\
\hline
\end{tabular}

"Significance of Spearman's rank correlation coefficient at 1 per cent level. to the unstretched length of muscle tissue (a longer muscle contains more cells in series, each with a similar individual contraction velocity; the individual contraction velocities add up to yield the contraction velocity of the entire muscle), the circumference of the bladder at capacity was considered to be proportional to the unstretched muscle length, and the parameter vmax was normalized by dividing it by this length. The parameter $U$ theoretically does not depend on the unstretched length of the muscle. It was, however, shown to be proportional to the stretched length. ${ }^{6}$ This dependence can be explained using the sliding-filament model of contracting muscle ${ }^{13}$ : stretching the muscle causes more overlap in the filaments and therefore more active sites. For this reason the parameter $U$ also was normalized by dividing it by the bladder circumference at capacity.

Spearman's rank correlation coefficients were calculated to investigate the associations among the parameters calculated from pressure-flow studies and isometric contractions, both for parameters normalized with respect to initial length and for unnormalized parameters.

\section{Results}

Table I shows averages and standard deviations of the calculated parameters for the entire group of 86 patients. Table II shows that the parameters vmax and $U$ are correlated significantly with the bladder volume at capacity, as expected. The other parameters, pmax and $w$, are neither expected nor found to be correlated 
TABLE III. Correlations between contractility parameters determined from pressure-flow studies and $U$ and $U / L$. Contractility parameters determined from isometric contractions immediately preceding pressure-flow studies. Correlations shown for both entire group of patients and subgroups of male and female children and male and female adults separately

\begin{tabular}{|c|c|c|c|c|c|c|c|c|c|c|}
\hline \multirow[b]{2}{*}{ Parameter } & \multicolumn{5}{|c|}{ Correlation With Parameter U- } & \multicolumn{5}{|c|}{ Correlation With Parameter U/L } \\
\hline & $\begin{array}{c}\text { Total } \\
(\mathrm{n}=86)\end{array}$ & $\begin{array}{c}\text { Boys } \\
(\mathrm{n}=27)\end{array}$ & $\begin{array}{c}\text { Girls } \\
(\mathrm{n}=34)\end{array}$ & $\begin{array}{c}\text { Men } \\
(\mathrm{n}=17)\end{array}$ & $\begin{array}{l}\text { Women } \\
(\mathrm{n}=8)\end{array}$ & $\begin{array}{c}\text { Total } \\
(\mathrm{n}=86)\end{array}$ & $\begin{array}{c}\text { Boys } \\
(\mathrm{n}=27)\end{array}$ & $\begin{array}{c}\text { Girls } \\
(\mathrm{n}=34)\end{array}$ & $\begin{array}{c}\text { Men } \\
(\mathrm{n}=17)\end{array}$ & $\begin{array}{r}\text { Women } \\
(\mathrm{n}=8)\end{array}$ \\
\hline$v \max$ & $0.33^{*}$ & 0.14 & $0.48^{*}$ & 0.26 & 0.62 & 0.18 & 0.05 & 0.42 & -0.08 & 0.62 \\
\hline $\operatorname{vmax} / \mathrm{L}$ & -0.02 & -0.11 & 0.08 & 0.14 & 0.33 & 0.13 & 0.01 & 0.32 & -0.03 & 0.33 \\
\hline $\operatorname{pmax}$ & 0.12 & 0.10 & -0.03 & $0.62 *$ & 0.07 & $0.27^{*}$ & 0.25 & 0.03 & $0.64^{*}$ & 0.07 \\
\hline $\mathrm{w}$ & $0.29 *$ & 0.32 & 0.21 & $0.67^{*}$ & 0.31 & $0.32^{*}$ & 0.39 & 0.22 & 0.44 & 0.31 \\
\hline
\end{tabular}

*Significance of Spearman's rank correlation coefficient at 1 per cent level

with volume. Table III shows correlations between the parameter $\mathrm{U}$ calculated from the isometric contractions, and both the normalized and the unnormalized parameters from the pressure-flow studies, both for the entire group of patients, and for the four subgroups of boys, girls, men, and women separately. The significant correlation between $U$ and vmax for the entire group is "caused" mainly by the two subgroups of females, both children and adults, whereas in adult males there is a significant correlation between $U$ and pmax. Table III also shows correlations between the normalized parameter $\mathrm{U} / \mathrm{L}$ and both the unnormalized and the normalized pressure-flow parameters. There is no significant correlation between $\mathrm{U} / \mathrm{L}$ and vmax, but there is an association with pmax and $w$, especially in adult males.

\section{Comment}

The values of the contractility parameters shown in Table I are considerably different from those published previously for a group of children. ${ }^{5}$ However, in that series ${ }^{5}$ a different method of calculation of contractility parameters was used, based on an assumed series elasticity. Errors in this assumption can cxplain the difference. The values of the normalized velocity parameters cannot be directly compared with those found in vitro, since the muscle length used for normalizing was not the initial muscle length, which is difficult to determine in a patient, but the bladder circumference at the maximum bladder capacity. From the average capacity (volume) in Table I, the average circumference can be calculated as approximately $25 \mathrm{~cm}$. If the value of the normalized parameter vmax/L is compared with the value found in vitro, ${ }^{7}$ it can be calculated that the actual average unstretched muscle length was about $10 \mathrm{~cm}$, which means that on average the patients' bladders were strained about 250 per cent at capacity.

Table III shows that there are significant correlations between contractility parameters based on isometric contractions $(\mathrm{U}, \mathrm{U} / \mathrm{L})$ and contractility parameters derived from pressureflow studies. Notice that the significant correlation between $\mathrm{U}$ and vmax is no longer significant when both parameters are normalized (Table III). This means that the former correlation is due mainly to the mutual dependence of both parameters on volume (Table II).

In males (especially adults) $U$ and $U / L$ are mainly related to the pressure parameter pmax. In females they are more closely related to the velocity parameter vmax. The reason for this sex-related difference is no doubt that the maximum contraction velocity vmax and the isometric pressure pmax are mutually exclusive realizations of the contractility of the muscle: the available energy can be used to generate either velocity or pressure. ${ }^{5}$ In male patients there is a substantial incidence of subvesical obstruction, and so high pressures are generated. Most females, however, micturate at low pressures, and so high contraction velocities are gencrated. The two rcalizations of the contractility of the detrusor are combined in the power parameter $\mathrm{w}$. The fact that $\mathrm{w}$ is related to $\mathrm{U}$ and $\mathrm{U} / \mathrm{L}$ to approximately the same degree in both males and females, whether children or adults, suggests that it is a universal pressureflow contractility parameter, suitable for both high- and low-pressure micturitions.

Since the overall correlations of $w$ (derived from pressure-flow studies) with $U$ and $U / L$ (derived from isometric contractions) are statistically significant, the two types of measurement give, at least partly, similar information about contractility of detrusor. However, the 
absolute values of the correlation coefficients are low, so that the information given is certainly not identical.

Both $\mathrm{U}$ and $\mathrm{U} / \mathrm{L}$ are approximately equally strongly related to $\mathrm{w}$. Since, however, $\mathrm{U}$ is dependent on bladder capacity, while $\mathrm{U} / \mathrm{L}$ is not, $\mathrm{U} / \mathrm{L}$ is more satisfactory as a contractility parameter.

To summarize, the parameter $\mathrm{U} / \mathrm{L}$ can be calculated from an isometric detrusor contraction. It is easy to measure, independent of bladder capacity and significantly correlated with measures of detrusor contractility derived from pressure-flow studies of micturition. It is, therefore, promising as a detrusor contractility parameter and deserves further study.

P.O. Box 1738

3000 DR Rotterdam, the Netherlands (DR. VAN MASTRIGT)

\section{References}

1. van Mastrigt $\mathrm{R}$, and van Duyl WA: Mechanics of detrusor contraction, determination of contractility, in Principles of Bladder Function and Urodynamics, Lameris, Utrecht, 1982, p 31.
2. Welkowitz W: Indices of cardiac status, IEEE Trans Biomed Eng 28-8: 553 (1981).

3. Hill AV: The heat of shortening and the dynamic constants of muscle, Proc Roy Soc London B 126: 136 (1938).

4. van Mastrigt $R$ : The on-line determination of the maximal contraction velocity of the urinary bladder from an isometric contraction, in Female Incontinence, New York, Alan R. Liss Inc., 1981, p 307.

5. IDEM: Determination of the contractility of children's bladders from isometric contractions, Urol Int 38: 354 (1983).

6. van Mastrigt R, and Glerum JJ: Electrical stimulation of smooth muscle strips from the urinary bladder of the pig, J Biomed Eng, 7: 2 (1985).

7. IDEM: In vitro comparison of isometric and stop-test contractility parameters for the 11rinary bladder, Urol Res 13: 11 (1985).

8. Griffiths DJ, and van Mastrigt R: The routine assessment of detrusor contraction strength, Neurourol Urodyn 4: 77 (1985)

9. Griffiths DJ, and Scholtmeijer RJ: Precise urodynamic assessment of anatomic urethral obstruction in boys, ibid 1: 97 (1982).

10. van Mastrigt R: A computer program for on-line measurement, storage, analysis and retrieval of urodynamic data, Comp Prog Biomed 18: 109 (1984).

11. van Mastrigt R, Thon HR, and van de Giessen $H$ : Analog data collection via a telephone line, a microprocessor-bascd, rcmote-controlled, data-acquisition system, Med Biol Eng Comp 21: 229 (1983).

12. van Mastrigt $R$, and Griffiths DJ: The contractility of the urinary bladder, Urol Int 34: 410 (1979).

13. Huxley AF: Review lecture: muscular contraction, J Phys 243: 1 (1974) 